\title{
Probing the infrared quark mass from highly excited baryons
}

\author{
P. Bicudo ${ }^{1}$, M. Cardoso ${ }^{1}$, T. Van Cauteren ${ }^{2}$, Felipe J. Llanes-Estrada ${ }^{3}$ \\ ${ }^{1}$ Instituto Superior Técnico, Lisboa, Portugal; ${ }^{2}$ Dept. Sub. and Rad. Phys., \\ Ghent University, Belgium; ${ }^{3}$ Dept. Física Teorica I, Univ. Complutense, Madrid, Spain
}

\begin{abstract}
We argue that three-quark excited states naturally group into quartets, split into two parity doublets, and that the mass splittings between these parity partners decrease higher up in the baryon spectrum. This decreasing mass difference can be used to probe the running quark mass in the mid-infrared power-law regime. A measurement of masses of high-partial wave $\Delta^{*}$ resonances should be sufficient to unambiguously establish the approximate degeneracy. We test this concept with the first computation of excited high-j baryon masses in a chirally invariant quark model.
\end{abstract}

Quantum Chromodynamics (QCD) has been thoroughly tested in high-energy physics, through hadron jets, Drell-Yan processes, $e^{-} e^{+}$annihilation, and deep inelastic scattering. Low-energy QCD manifests chiral symmetry breaking $(\chi \mathrm{SB})$ that enhances quark masses in the infrared (IR), generating most of the visible mass in our universe, and that removes the degeneracy from the ground states of light hadron spectra (so light hadrons do not come in parity doublets). The excited lightquark baryon spectrum, with masses above that of the $\Delta$-resonance $(1232 \mathrm{MeV})$, has not been accessible to any form of perturbation theory, effective or fundamental.

Insensitivity to chiral symmetry breaking, recently stressed by Glozman [1, 2, 3], and retrospectively present in the excited meson spectra computed in chirally invariant quark models [4, 5] has led many hadron physicists to accept that spontaneous $\chi \mathrm{SB}$, the salient feature of the low hadron spectrum, actually becomes less important for highly excited resonances. For these, chiral symmetry is asymptotically realized in Wigner mode, arranging hadrons in degenerate chiral multiplets [6, 7]. We have realized that a formalization of this statement is that the ratio of the quark mass to its momentum $\left\langle\frac{m}{k}\right\rangle$ provides a new perturbative parameter to study some aspects of the excited hadron spectrum when the typical momentum becomes higher than the running mass. Reasoning in reverse, we propose employing a cancellation in mass differences of very excited resonances, to access this small parameter, and hence the quark mass. We also show analytically and numerically that three-quark states naturally group into quartets with two states of each parity. Diagonalizing the chiral charge in terms of quarks, the quartet is split into two parity doublets, and all mass splittings tend to decrease when going higher in the spectrum. For simplicity and to minimize the impact of molecular meson-nucleon configurations [8], we study the maximum-spin excitations $\Delta^{*}$ of the Delta baryon.

The $u$ or $d$ quark mass [9] (understood as taken from the full two-point function of the field theory), is theorized to vary from $m \simeq 1-6 \mathrm{MeV}$ at a high, perturbative momentum $k$ scale (typically $\gg \Lambda_{\mathrm{QCD}} \sim 210 \mathrm{MeV}$ ), to a constituent mass of circa $300 \mathrm{MeV}$ at vanishing momentum $k \simeq \Lambda_{Q C D}$. This IR enhancement of two orders of

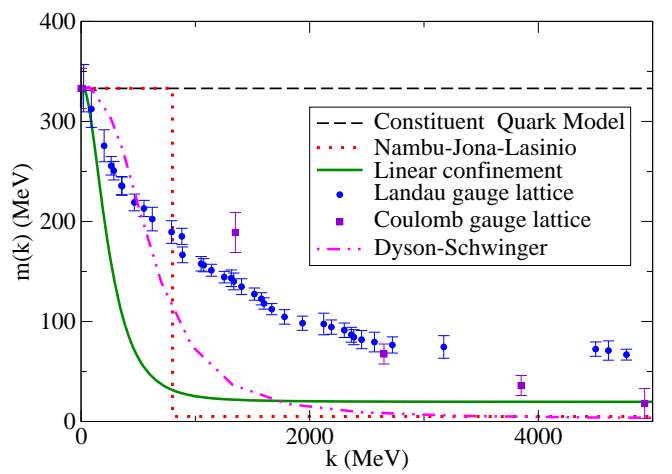

FIG. 1: IR enhancement of the light quark mass due to spontaneous $\chi \mathrm{SB}$. Shown are quark masses in the main approaches to QCD, all multiplied by an arbitrary factor to match them at quark momentum norm $k \rightarrow 0$. (References in the text.)

magnitude is shown in Fig. 1. The simplest constituent quark model considers a constant mass [10], without the characteristic step-function of the Nambu and JonaLasinio model (also shown). Breaking chiral symmetry with a linear potential forces a power-law $m \rightarrow \frac{C}{k^{4}}$ for $m(k) \ll k$. Lattice QCD [11] and Schwinger-Dyson equations [12], both Euclidian approaches to QCD, also show power-law decays of the light quark mass. However little has been said about this experimentally, for $k \sim 1.5$ $\mathrm{GeV}$, where we already have $m(k) \ll k$.

In Hamiltonian dynamics, the quark mass appears in the Dirac spinors $U_{\kappa \lambda}$ that govern the quark-quark interaction. In the heavy quark limit, spin-tensor potentials have been successfully derived by expanding in orders of $k / m(k)$. For light quarks the opposite, ultrarelativistic limit of $k \gg m(k)$ is relevant. Spinors are then conveniently expanded in the inverse ratio $m(k) / k$, or

$$
\begin{array}{r}
U_{\kappa \lambda}=\frac{1}{\sqrt{2 E(k)}}\left[\begin{array}{c}
\sqrt{E(k)+m(k)} \chi_{\lambda} \\
\sqrt{E(k)-m(k)} \vec{\sigma} \cdot \hat{\kappa} \chi_{\lambda}
\end{array}\right] \\
\underset{k \rightarrow \infty}{\longrightarrow} \frac{1}{\sqrt{2}}\left[\begin{array}{c}
\chi_{\lambda} \\
\vec{\sigma} \cdot \hat{\kappa} \chi_{\lambda}
\end{array}\right]+\frac{1}{2 \sqrt{2}} \frac{m(k)}{k}\left[\begin{array}{c}
\chi_{\lambda} \\
-\vec{\sigma} \cdot \hat{\kappa} \chi_{\lambda}
\end{array}\right]
\end{array}
$$

with $E(k)=\sqrt{k^{2}+m(k)^{2}}$, having kept the leading chirally invariant term, and a next order chiral symmetry breaking $\frac{m(k)}{k}$ term. Non-chiral, spin-dependent potentials in the quark-quark interaction originate from the 
second term in an expansion of $H^{\mathrm{QCD}}[13]$ in the weak sense (that is, not of the Hamiltonian operator itself, but a restriction thereof to the Hilbert space of highly excited resonances, where $\langle k\rangle$ is large):

$$
\left\langle n\left|H^{Q C D}\right| n^{\prime}\right\rangle \simeq\left\langle n\left|H_{\chi}^{Q C D}\right| n^{\prime}\right\rangle+\left\langle n\left|\frac{m(k)}{k} H_{\chi}^{Q C D^{\prime}}\right| n^{\prime}\right\rangle+\ldots
$$

To access the quark mass from experiment one can exploit the approximate parity degeneracy, that would be exact in the presence of only the first term $H_{\chi}^{Q C D}$, of excited baryons. This degeneracy follows from invariance under transformations generated by the chiral charge 14] $Q_{5}^{a}=\int d \mathbf{x} \psi^{\dagger}(x) \gamma_{5} \frac{\tau^{a}}{2} \psi(x)$ and $\left[Q_{5}^{a}, H\right]=0$. However Chiral Symmetry is spontaneously broken by the ground state, $Q_{5}^{a}|0\rangle \neq 0$ providing a large quark mass in the propagator, $m(k)$, pseudo-Goldstone bosons $(\pi, K, \eta)$, and the loss of parity degeneracy in ground state baryons. Substituting the spinors, and in terms of Bogoliubovrotated $q \bar{q}$ normal modes $B$ and $D, Q_{5}$ becomes

$$
\begin{gathered}
Q_{5}^{a}=\int \frac{d^{3} k}{(2 \pi)^{3}} \sum_{\lambda \lambda^{\prime} f f^{\prime} c}\left(\frac{\tau^{a}}{2}\right)_{f f^{\prime}} \frac{k}{\sqrt{k^{2}+m^{2}(k)}}(3) \\
\left((\boldsymbol{\sigma} \cdot \hat{\mathbf{k}})_{\lambda \lambda^{\prime}}\left(B_{k \lambda f c}^{\dagger} B_{k \lambda^{\prime} f^{\prime} c}+D_{-k \lambda^{\prime} f^{\prime} c}^{\dagger} D_{-k \lambda f c}\right)+\right. \\
\left.\frac{m(k)}{k}\left(i \sigma_{2}\right)_{\lambda \lambda^{\prime}}\left(B_{k \lambda f c}^{\dagger} D_{-k \lambda^{\prime} f^{\prime} c}^{\dagger}+B_{k \lambda^{\prime} f^{\prime} c} D_{-k \lambda f c}\right)\right)
\end{gathered}
$$

In the presence of Spontaneous $\chi \mathrm{SB}, m(k) \neq 0$, and the last term realizes chiral symmetry non-linearly in the spectrum as it creates/destroys a pion. But when $\langle k\rangle \gg m(k)$, the $\boldsymbol{\sigma} \cdot \hat{\mathbf{k}}$-term dominates and chiral symmetry is realized linearly (with only quark counting operators flipping parity and spin). This happens for a highly excited baryon resonance whose constituents have a momentum distribution peaked at higher momenta than the IR enhancement of $m(k)$ (Fig. 1).

For the ground-state resonances in excited spin channels, three-quark variational wavefunctions are relevant and a reasonable phenomenological guide. When $Q_{5}$ acts on such wavefunction $|N\rangle=F_{i j k} B_{i}^{\dagger} B_{j}^{\dagger} B_{k}^{\dagger}|\Omega\rangle$, the result also contains three quarks, but one of them is spinrotated from $B_{k \lambda}$ to $\sigma \cdot \hat{k}_{\lambda \lambda^{\prime}} B_{k \lambda^{\prime}}$. Successive application of the chiral charge spin-rotates further quarks, changing each time the parity of the total wavefunction. However the sequence of states is closed since $\sigma \cdot \hat{k} \sigma \cdot \hat{k}=\mathbb{I}$. In fact, starting with an arbitrary spin-symmetric wavefunction with parity $P$, one generates our claimed Baryon Quartet of equal isospin (isospin index dropped):

$$
\begin{array}{r}
\left|N_{0}^{P}\right\rangle=\sum F_{i j k}^{P} B_{i}^{\dagger} B_{j}^{\dagger} B_{k}^{\dagger}|\Omega\rangle \\
\left|N_{1}^{-P}\right\rangle=\frac{1}{3} \sum F_{i j k}^{P}\left(\left(\boldsymbol{\sigma} \cdot \hat{\mathbf{k}} B^{\dagger}\right)_{i} B_{j}^{\dagger} B_{k}^{\dagger}+\right. \\
\left.B_{i}^{\dagger}\left(\boldsymbol{\sigma} \cdot \hat{\mathbf{k}} B^{\dagger}\right)_{j} B_{k}^{\dagger}+B_{i}^{\dagger} B_{j}^{\dagger}\left(\boldsymbol{\sigma} \cdot \hat{\mathbf{k}} B^{\dagger}\right)_{k}\right)|\Omega\rangle
\end{array}
$$

$$
\begin{array}{r}
\left|N_{2}^{P}\right\rangle=\frac{1}{3} \sum F_{i j k}^{P}\left(\left(\boldsymbol{\sigma} \cdot \hat{\mathbf{k}} B^{\dagger}\right)_{i}\left(\boldsymbol{\sigma} \cdot \hat{\mathbf{k}} B^{\dagger}\right)_{j} B_{k}^{\dagger}+\right. \\
\left.B_{i}^{\dagger}\left(\boldsymbol{\sigma} \cdot \hat{\mathbf{k}} B^{\dagger}\right)_{j}\left(\boldsymbol{\sigma} \cdot \hat{\mathbf{k}} B^{\dagger}\right)_{k}+\left(\boldsymbol{\sigma} \cdot \hat{\mathbf{k}} B^{\dagger}\right)_{i} B_{j}^{\dagger}\left(\boldsymbol{\sigma} \cdot \hat{\mathbf{k}} B^{\dagger}\right)_{k}\right)|\Omega\rangle \\
\left|N_{3}^{-P}\right\rangle=\sum F_{i j k}^{P}\left(\boldsymbol{\sigma} \cdot \hat{\mathbf{k}} B^{\dagger}\right)_{i}\left(\boldsymbol{\sigma} \cdot \hat{\mathbf{k}} B^{\dagger}\right)_{j}\left(\boldsymbol{\sigma} \cdot \hat{\mathbf{k}} B^{\dagger}\right)_{k}|\Omega\rangle
\end{array}
$$

which is a natural basis to discuss chiral symmetry restoration in baryons, through wavefunctions that are linear combinations $|N\rangle=\sum c_{a}\left|N_{a}\right\rangle$. This reducible representation of $Q_{5}^{a=3}$ corresponds to $[(3 / 2,0) \oplus(0,3 / 2)]^{2}$ of $S U(2)_{L} \times S U(2)_{R}$, and can be embedded in larger ones including nucleons [7] .

Because $\left[Q_{5}, H\right]=0$, the two can be diagonalized simultaneously. The representation of the chiral charge in the (non-orthonormal) quartet coordinates breaks in two blocks since $Q_{5}$ changes parity, or using the square charge

$$
Q_{5}^{2}\left(\begin{array}{l}
c_{0} \\
c_{2}
\end{array}\right)=\left[\begin{array}{ll}
3 & 2 \\
6 & 7
\end{array}\right]\left(\begin{array}{l}
c_{0} \\
c_{2}
\end{array}\right) .
$$

Immediately one sees that the two linear combinations $N_{0}-N_{2}$ and $N_{0}+3 N_{2}$ diagonalize the square chiral charge in the positive parity sector (if the original wavefunction had positive parity), with $N_{1}-N_{3}$ and $3 N_{1}+N_{3}$ doing so for negative parity. The quartet then separates into two doublets connected by the chiral charge

$Q_{5}\left(N_{0}-N_{2}\right)=N_{1}-N_{3} \quad Q_{5}\left(N_{0}+3 N_{2}\right)=3\left(3 N_{1}+N_{3}\right)$

$\left.Q_{5}\left(N_{1}-N_{3}\right)=N_{0}-N_{2} \quad Q_{5}\left(3 N_{1}+N_{3}\right)=3\left(N_{0}+3 N_{2}\right) 6\right)$

Two doublets appear with different eigenvalues of $Q_{5}^{2}, 1$ and 9 respectively, and the interdoublet splitting becomes a dynamical question (we will argue shortly that it is small for highly excited baryons). However, the splitting within the doublet must vanish asymptotically. Even for fixed (not running) quark mass, when the typical kinetic energy is high enough $\langle k\rangle>>m$, the effects of the quark mass are negligible. Parity doubling then boils down to whether the interaction terms are also chiral symmetry violating or not.

We now exploit the smallness of the $\left|M^{P=+}-M^{P=-}\right|$ mass difference with increasing angular momentum to guide experiment in obtaining information about the running quark mass. For this we establish how the $j$ dependence of the splitting is related to the $k$-dependence of $m(k)$. The $M^{ \pm}$in our proposed study are the masses of the ground state quartets of the $\Delta$ spectrum, with parity \pm and in the limit of large total angular momentum $j>>3 / 2$. The two approximately degenerate masses $M^{+}$and $M^{-}$are in the same leading linear Regge trajectory, phenomenologically fixing their $j$-dependence to

$$
j=\alpha_{0}+\alpha M^{ \pm 2} \underset{j \rightarrow \infty}{\longrightarrow} \alpha M^{ \pm^{2}} .
$$

The parity of the ground state alternates between + and - as the angular momentum steps up by one. Large $j$ 


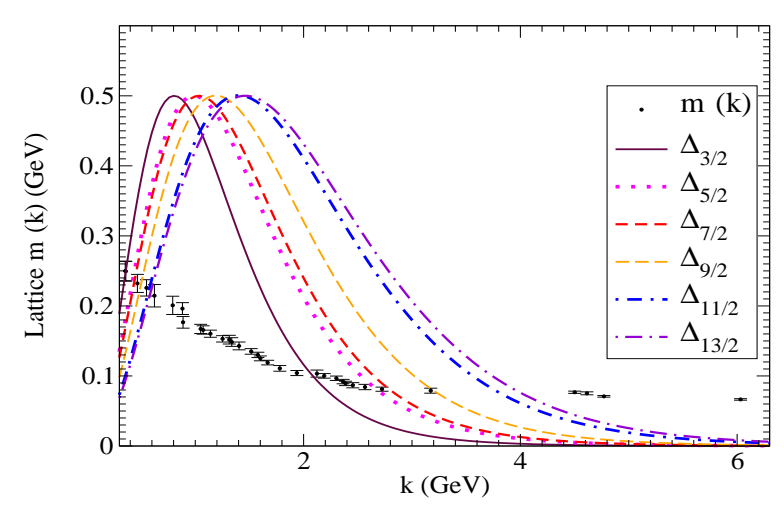

FIG. 2: Typical momentum distributions of increasingly excited $\Delta_{3 / 2}, \ldots \Delta_{13 / 2}$ resonances overlap less and less with the dynamically generated IR quark mass so that $\left\langle n\left|\frac{m(k)}{k} H_{\chi}^{Q C D^{\prime}}\right| n\right\rangle$ becomes small. (Illustrative variational wavefunctions for a linear potential with string tension $\sigma=$ $0.135 \mathrm{GeV}^{2}$, not normalized for visibility).

is equivalent to large quark orbital angular momentum $l \gg 1$ since the spin is finite, and also to a large average linear momentum $\langle k\rangle \gg \Lambda_{Q C D}$. Figure 2 shows how the wavefunctions of high- $j$ states overlap with the running $m(k)$. In these states $\langle m(k) / k\rangle$ is a useful perturbative parameter. From the relativistic virial theorem [15] between kinetic and total energies,

$$
\langle k\rangle \rightarrow c_{2} M^{ \pm} \rightarrow \frac{c_{2}}{\sqrt{\alpha}} \sqrt{j}
$$

(where $c_{2}$ is a constant; for instance in the case of a linear potential model, and 3 quarks in a baryon, $c_{2}=1 / 6$ ).

The first term in Eq. (2) cancels out in the difference $\left|M^{+}-M^{-}\right|<<M^{ \pm}$(while both $M^{ \pm}$are dominated by the chirally invariant term, their difference stems from the dynamically generated quark mass) thus exposing the second term in eq.(2), proportional to $\left\langle\frac{m(k)}{k}\right\rangle$, viz.

$$
\left|M^{+}-M^{-}\right| \rightarrow\left\langle\frac{m(k)}{k} H_{\chi}^{Q C D^{\prime}}\right\rangle \rightarrow c_{3} \frac{m(\langle k\rangle)}{\langle k\rangle}\left\langle H_{\chi}^{Q C D^{\prime}}\right\rangle
$$

(the factorization is allowed by the mean-value theorem at the price of a constant that we do not attempt to determine here). This equation is analogous to the renowned Gell-Mann-Oakes-Renner relation $M_{\pi}^{2}=-m_{q} \frac{\langle\bar{\psi} \psi\rangle}{f_{\pi}^{2}}$ but active when chiral symmetry is realized linearly, as in the high-baryon excitations we examine.

Notice that $H_{\chi}^{Q C D}$ ' differs from $H_{\chi}^{Q C D}$ due to the sign of the $(\boldsymbol{\sigma} \cdot \hat{\mathbf{k}})_{i}$ term in the $\langle m(k) / k\rangle$ expansion of the quark $i$ spinor in eq. (2). This changes the sign of spindependent terms in $H_{\chi}^{Q C D^{\prime}}$, led in high $j$ by the spinorbit term. In $H_{\chi}^{Q C D}$ the spin-orbit is crucial to correct the angular momentum in the centrifugal barrier term from $\mathbf{L}_{i}{ }^{2}$ to the chirally invariant $\mathbf{L}_{i}{ }^{2}+2 \mathbf{L}_{i} \cdot \mathbf{S}_{i}=\mathbf{J}_{i}{ }^{2}-\frac{3}{4}$. But the opposite sign of the spin-orbit term of $H_{\chi}^{Q C D}{ }^{\prime}$ produces a $\left|M^{+}-M^{-}\right|$mass difference, enhanced in the IR by $\langle m(k) / k\rangle$. Since the centrifugal barrier scales in

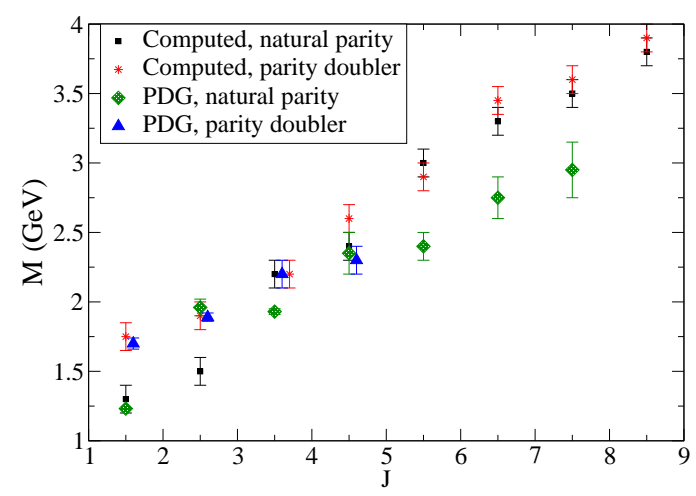

FIG. 3: Parity doubling in the spin-excited $\Delta$ spectrum for a string tension $\sigma=0.135 \mathrm{GeV}^{2}$ and the mass gap angle matched to the lattice data. A $q q q$ variational Monte-Carlo computation of the masses with a chiral Hamiltonian shows that the ground-states with parity \pm for each $j=3 / 2 \ldots 13 / 2$ quickly degenerate. Experimentally the degeneracy can be claimed for the $9 / 2$ states only.

the large $j$ limit like the baryon mass $M^{ \pm}$, and the spinorbit term comes with one power of $j$ less than it,

$$
\left\langle H_{\chi}^{Q C D^{\prime}}\right\rangle \rightarrow c_{5} M^{ \pm} j^{-1} \rightarrow \frac{c_{5}}{\sqrt{\alpha}} \sqrt{\frac{1}{j}} .
$$

Combining the $j$-power-law analysis of eqs. (77) to (10),

$$
\left|M^{+}-M^{-}\right| \rightarrow \frac{c_{3} c_{5}}{c_{2} \sqrt{\alpha}} m(\langle k\rangle) j^{-1}
$$

that links the IR enhancement of the quark mass to baryon spectroscopy in a usable way. An experimental extraction proceeds by just fitting the exponent $-i$ of $j$ in the splitting $\left|M^{+}-M^{-}\right| \propto j^{-i}$. Then, we obtain,

$$
m\left(\frac{c_{2}}{\sqrt{\alpha}} \times \sqrt{j}\right) \propto j^{-i+1} \text { and } m(k) \propto k^{-2 i+2} .
$$

The same exponent $i$ in eq. (12) can be obtained from the fit [16] to the $\left|M^{+}-M^{-}\right|$with increasing $j$ ! Though the quark mass itself is gauge-dependent, our analysis suggests that its power-law exponent is not, since it is directly related to an observable.

We next contribute the first competitive chirally invariant quark model computation of parity doubling in the excited baryon spectrum, shown in figure 3 , employing a well-known model of Coulomb-QCD that can be seen as a field theory upgrade of the Cornell model,

$$
\begin{aligned}
H & =-g_{s} \int d \mathbf{x} \Psi^{\dagger}(x) \alpha \cdot \mathbf{A}(x) \Psi(x)+T r \int d \mathbf{x}(\mathbf{E} \cdot \mathbf{E}+\mathbf{B} \cdot \mathbf{B}) \\
& +\int d \mathbf{x} \Psi_{q}^{\dagger}(-i \alpha \cdot \nabla+\beta m) \Psi_{q}-\frac{1}{2} \int d \mathbf{x} d \mathbf{y} \rho_{\mathbf{x}}^{a} V_{L}(|\mathbf{x}-\mathbf{y}|) \rho_{\mathbf{y}}^{a}
\end{aligned}
$$

with the kernel $V_{L}(r)=\sigma r$ with string tension $\sigma=0.135$ $\mathrm{GeV}^{2}$, coupled to the color charge density $\rho^{a}(\boldsymbol{x})=$ $\Psi^{\dagger}(\boldsymbol{x}) T^{a} \Psi(\boldsymbol{x})+f^{a b c} \mathbf{A}^{b}(\boldsymbol{x}) \cdot \boldsymbol{\Pi}^{c}(\boldsymbol{x})$. We solve the BCS gap equation and employ both the so calculated $m(k)$ 

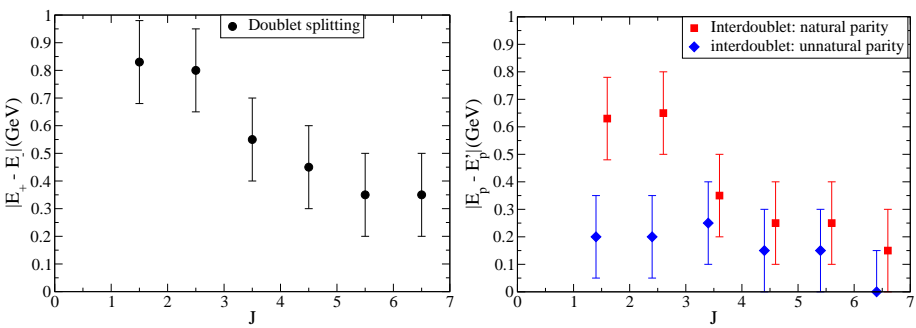

FIG. 4: All model quartet splittings decrease for high $j$. Left: intradoublet splitting for the first parity doublet. Right: interdoublet splittings between natural and unnatural parity states. The latter is smaller for low $j$.

quark mass as well as lattice computation in Landau and Coulomb gauges 11. The model has the same chiral structure of QCD[4], satisfying the Gell-MannOakes-Renner relation and other low-energy theorems, and allowing computations of static $N \pi$ observables [17]. Pauli antisymmetrization of wavefunctions, spin summations and the 9-dimensional integrations are performed in a computer program, employing Monte-Carlo integration [18], to compute the excited $\Delta^{*}$ spectrum. We proceed variationally and employ several types of wavefunctions. While a suboptimal variational basis for low-lying states, the quartet states coincide with $H$-eigenstates high in the spectrum. We use both the quartet and the fixed $L$ variational basis.

As can be seen from figure 4, within our variational and Monte-Carlo integration errors, the model splittings drop with $j$, as predicted analytically; a model statement equivalent to the generic eq. (9) reads

$$
\begin{array}{r}
M_{+}-M_{-}=3 \int \frac{d^{3} k_{1}}{(2 \pi)^{3}} \frac{d^{3} k_{2}}{(2 \pi)^{3}}\left(\frac{2}{3}\right) \int \frac{d^{3} q}{(2 \pi)^{3}} \\
\hat{V}(q) \frac{1}{2}\left(\frac{m\left(\left|\mathbf{k}_{1}\right|\right)}{\left|\mathbf{k}_{1}\right|}+\frac{m\left(\left|\mathbf{k}_{1}+\mathbf{q}\right|\right)}{\left|\mathbf{k}_{1}+\mathbf{q}\right|}\right) F^{* \lambda_{1} \lambda_{2} \lambda_{3}}\left(\mathbf{k}_{1}, \mathbf{k}_{2}\right) \\
\left(\mathbb{I}-\sigma \hat{\mathbf{k}}_{1} \sigma \widehat{\mathbf{k}_{1}+\mathbf{q}}\right)_{\lambda_{1} \mu_{1}} F^{\mu_{1} \lambda_{2} \lambda_{3}}\left(\mathbf{k}_{1}+\mathbf{q}, \mathbf{k}_{2}-\mathbf{q}\right)
\end{array}
$$

If precise data becomes available at ELSA or Jefferson Lab for the $\Delta_{J}$ with $J=7 / 2,9 / 2,11 / 2,13 / 2 \cdots$ parity quartets, one should be able to distinguish between the $1 / j$ fall of $\left|M^{+}-M^{-}\right|$for non-chiral models (with a constant difference $\left(M^{+}-M^{-}\right)^{2}$ between the Regge Trajectories [10]), and the faster drops for chiral theories such as QCD. Since the two doublets are closely degenerate, both positive and negative parity ground states will have a nearby resonance with identical quantum numbers. Given their width, it is likely they will only be distinguished by very careful exclusive decay analysis.

Further, if the $\Delta$ spectrum could be measured up to high resonance masses, beyond the IR $m(k)$ enhancement, and a lattice calculation of $\left\langle H_{\chi}^{Q C D^{\prime}}\right\rangle$ became available, an almost direct measurement of the current quark mass would follow in a regime of logarithmic, rather than power-law running. We advocate that a measurement of masses of high partial-wave $\Delta$ resonances with an accuracy of $50 \mathrm{MeV}$ should suffice to establish the approximate degeneracy and test the concept of running quark mass in the IR. The presented concepts should also further motivate analysis of empirical data [19] such as EBAC (Excited Baryon Analysis Center) at Jefferson Lab as well as Lattice QCD collaborations who are addressing the baryon spectrum.

We thank L. Glozman for useful conversations, and grants FPA 2008-00592/FPA, FIS2008-01323, CERN/FP /83582/2008, POCI/FP /81933/2007, /81913/2007, PDCT/FP /63907/2005 and /63923/2005, Spain-Portugal billateral grant HP2006-0018 / E-56/07, as well as the Scientific Research Fund of Flanders.

[1] L. Y. Glozman, Phys. Lett. B 475 (2000) 329.

[2] E. S. Swanson, Phys. Lett. B 582, 167 (2004).

[3] R. F. Wagenbrunn and L. Y. Glozman, Phys. Lett. B 643, 98 (2006); T. D. Cohen and L. Y. Glozman, Mod. Phys. Lett. A 21, 1939 (2006); L. Y. Glozman, A. V. Nefediev and J. E. F. Ribeiro, Phys. Rev. D 72, 094002 (2005).

[4] A. Le Yaouanc et al., Phys. Rev. D 31 (1985) 137.

[5] P. Bicudo et al., Phys. Lett. B 442, 349 (1998).

[6] C. E. DeTar and T. Kunihiro, Phys. Rev. D 39, 2805 (1989); D. Jido, T. Hatsuda and T. Kunihiro, Phys. Rev. Lett. 84, 3252 (2000).

[7] T. D. Cohen and L. Y. Glozman, Phys. Rev. D 65, 016006 (2001) arXiv:hep-ph/0102206.

[8] S. Sarkar et al., arXiv:0902.3150 [hep-ph].

[9] C. Amsler et al. [PDG], Phys. Lett. B 667, 1 (2008).

[10] J. Segovia, D. R. Entem and F. Fernandez, Phys. Lett. B 662, 33 (2008).

[11] P. O. Bowman, et al., Nucl. Phys. Proc. Suppl. 161, 27 (2006); M. B. Parappilly et al, Phys. Rev. D 73, 054504 (2006). S. Furui, arXiv:0801.0325 [hep-lat].

[12] R. Alkofer et al., Ann. Phys. NY (in press) arXiv:0804.3042 [hep-ph].

[13] For a discussion on $H^{\mathrm{QCD}}$, a good starting point is N. H. Christ and T. D. Lee, Phys. Rev. D22 (1980) 939.

[14] A. V. Nefediev, J. E. F. Ribeiro and A. P. Szczepaniak, JETP Lett. 87, 271 (2008).

[15] W. Lucha and F. F. Schoberl, Mod. Phys. Lett. A 5, 2473 (1990).

[16] For a linear potential, with $m(k) \propto k^{-4},\left|M^{+}-M^{-}\right| \propto$ $\frac{1}{j^{3}}$. If, on the contrary, the quark mass is constant, and still the potential remains chirally invariant, then the decrease follows a slower $j^{-1}$. We can retrospectively understand analytically the $q \bar{q}$ numerical results of [3], with chiral quartets and with the $j$-scaling of the splittings. While their doublet $\left|M^{+}-M^{-}\right| \sim \frac{1}{j^{3}}$; their interdoublet splitting follows a weaker $\frac{1}{j^{3 / 2}}$ as expected in a corollary of eq. (10) for spin-independent potentials.

[17] P. Bicudo, G. Krein and J. E. F. Ribeiro, Phys. Rev. C 64 (2001) 025202.

[18] T. Hahn, Comput. Phys. Commun. 168, 78 (2005).

[19] B. Julia-Diaz et al, Phys. Rev. C 77 (2008) 045205. 